\title{
Pasteurellosis and other respiratory bacterial infections
}

Glisson, John ; Hofacre, Charles; Christensen, Jens Peter

Published in:

Diseases of poultry

Publication date:

2013

Document version

Publisher's PDF, also known as Version of record

Citation for published version (APA):

Glisson, J., Hofacre, C., \& Christensen, J. P. (2013). Pasteurellosis and other respiratory bacterial infections. In D. E. Swayne (Ed.), Diseases of poultry (13 ed., pp. 807-823). Wiley-Blackwell. 

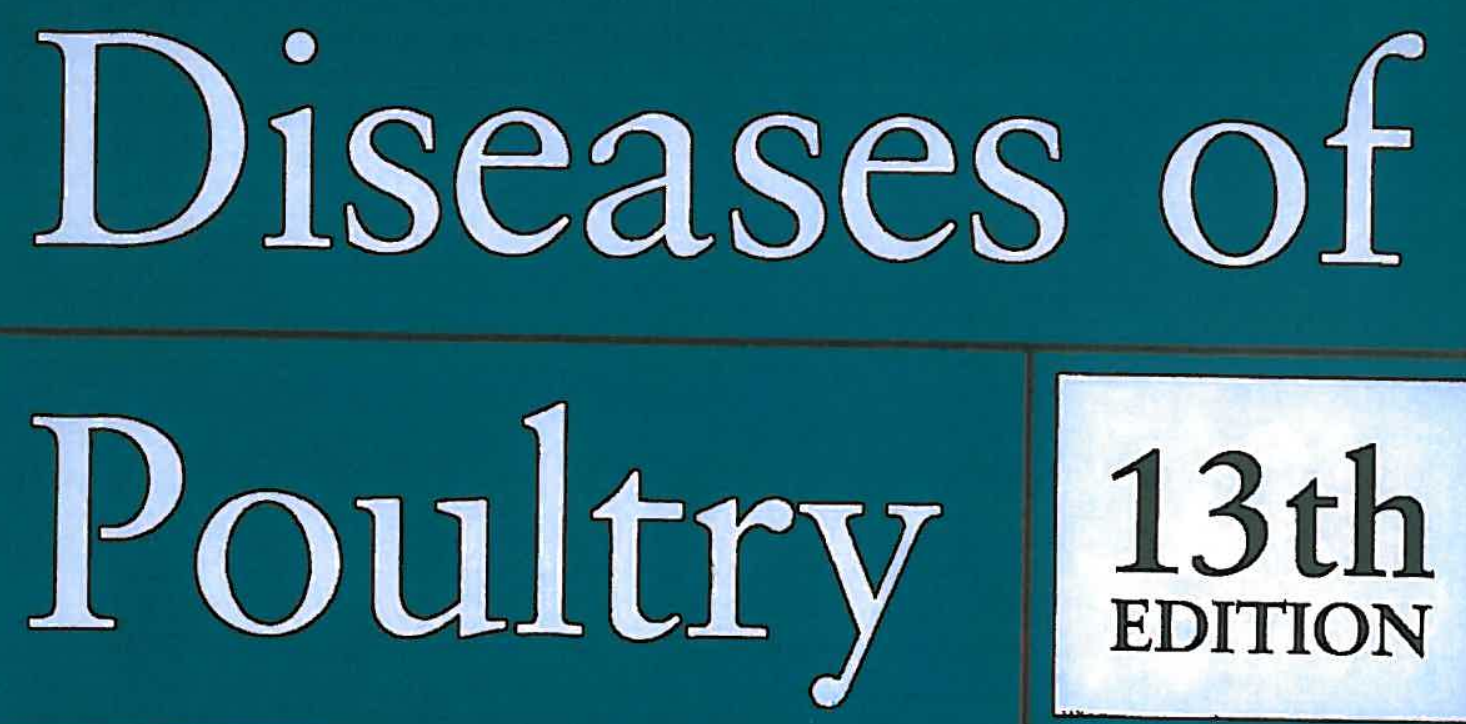

Editor in Chief: David E. Swayne Associate Editors: John R. Glisson, Larry R. McDougald, Lisa K. Nolan, David L. Suarez, and Venugopal Nair 

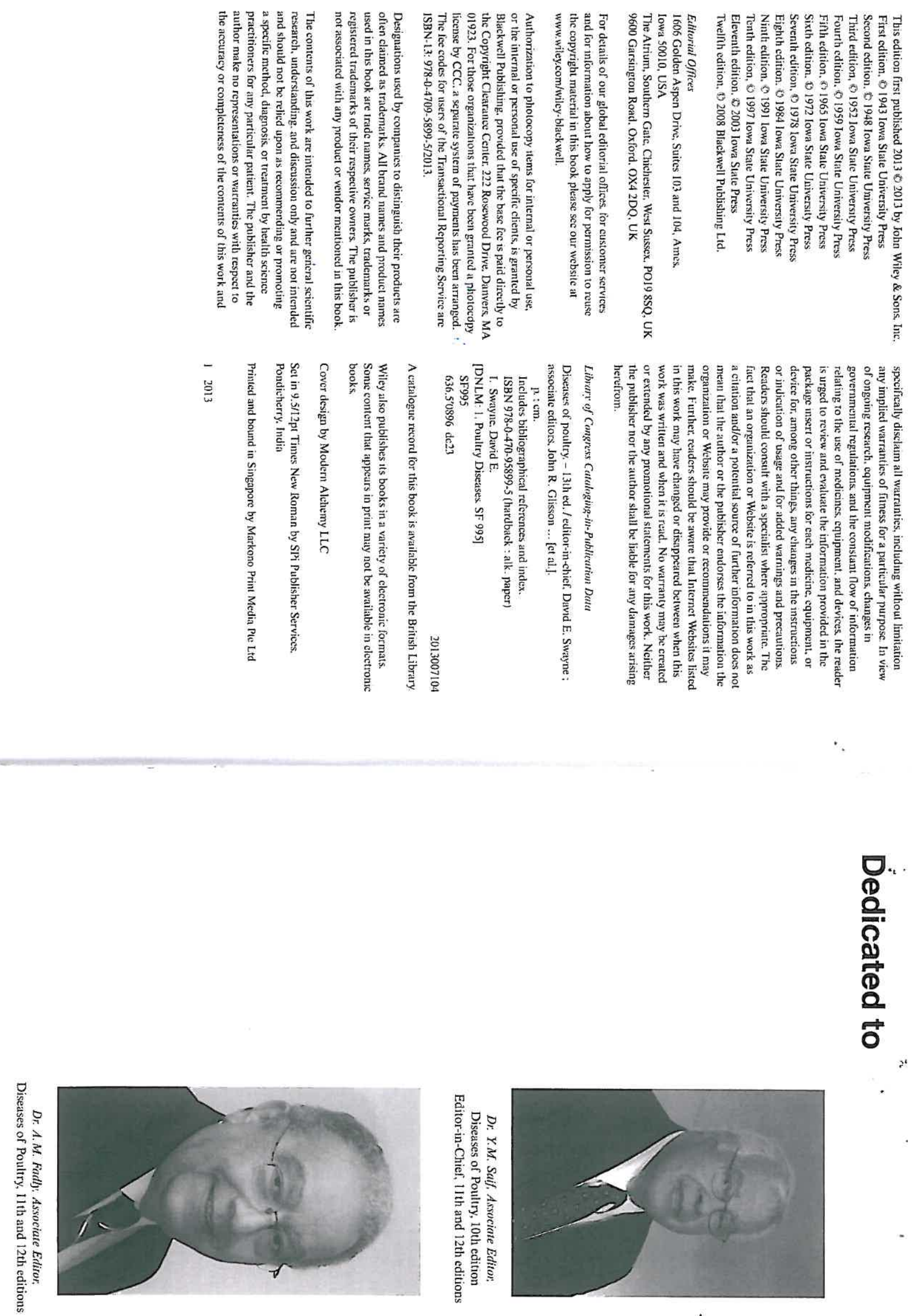

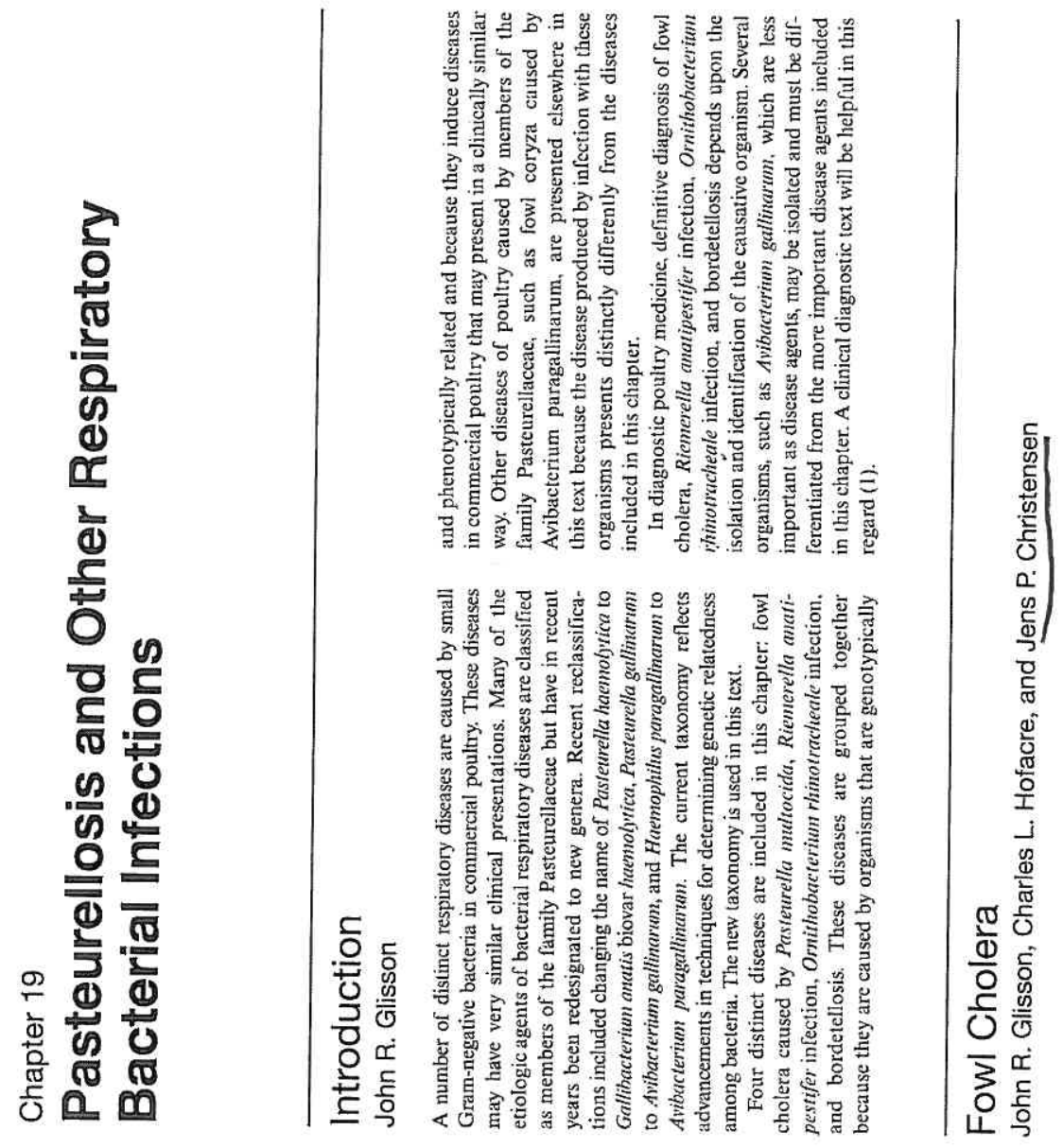

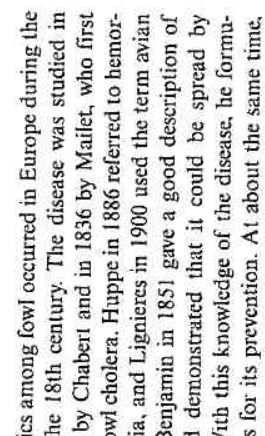

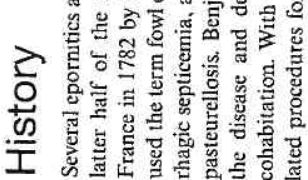

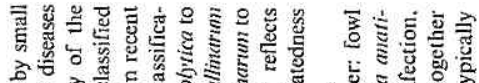

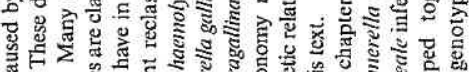

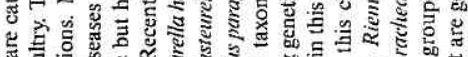

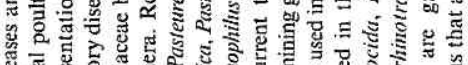

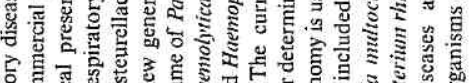

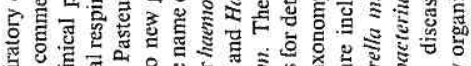

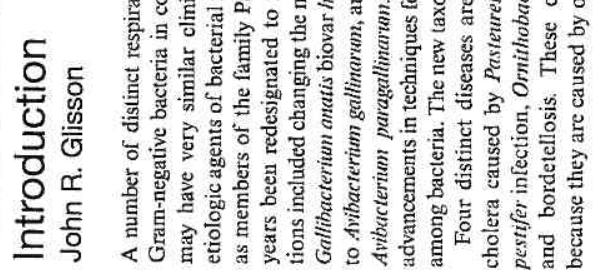

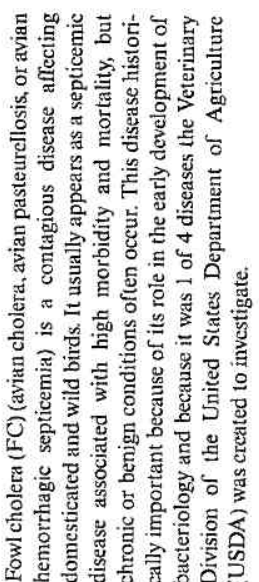


Renault, Reynal, and Delafond demonstrated its transmissibility to various species by inoculation. In 1877 and 1878 , Perroncito of Italy and Semmer of Russia observed in tissues of affected birds a bacterium that had a rounded form and occurred singly or in pairs. In 1879. Toussaint isolated the bacterium and proved it was the sole cause of the disease (52).

Pasteur (126) isolated the organism and grew pure cultures in chicken broth. In further studies, Pasteur $(127,128)$ used the FC organism to perform his classic experiments in attenuation of bacteria for use in producing immunity. Salmon (153) appears to have been the first to study the disease in the United States. A good description of disease signs was reported, however, as early as 1867 in Iowa, where losses of chickens, turkeys, and geese had occurred (4).

\section{Etiology}

\section{Classification}

Pasteurella multocida is the causative agent of FC. When pronouncing multocida, the accent should be on the "ci" (16) rather than on the "to" as given in the 7th and 8th editions of Bergey's Mamual. In the past, the bacterium has been given many names, including Micrococcus gallicidus, 1883; M. cholerae gallinarum, 1885; Octopsis cholerae gallinarum. 1885; Bacterium cholerae gallinarum, 1886; Bacillus cholerae gallinarum. 1886; P. cholerae-gallinarum, 1887; Coccobacillus avicidus, 1888; P. avicida, 1889; Bacterium multicidum, 1899; P. avitm, 1903; Bacillus ariseptictus, 1903: Bacterium avisepticum, 1903; Bacterium avisepticus, 1912; and $P$. ariseptica, $1920(16,19)$.

For a while, each isolate of $P$. multocida was named according to the animal from which it was isolated, such as $P$. avicida or $P$. aviseptica, $P$. nuricida or $P$. nuriseptica. In 1929, it was suggested that all isolates be referred to as $P$. septica (170). This name was used mainly in the United Kingdom and can be found in recent literature. Pasteurella multocidla, proposed by Rosenbusch and Merchant (151), is now accepted as the official name in Bergey's Manual and is used exclusively throughout the world.

\section{Morphology and Staining}

P. multocida is a Gram-negative, nonmotile, nonspore-forming rod occurring singly, in pairs, and occasionally as chains or filaments. It measures $0.2-0.4 \times 0.6-2.5 \mathrm{~mm}$ but tends to become pleomorphic after repeated subculture. A capsule can be demonstrated in recently isolated cultures using indirect methods of staining (Figure 19.1). In tissues, blood, and recently isolated cultures, the organism stains bipolar (Figure 19.2). Pili have been reported $(49,135)$.

\section{Growth Requirements}

P. multocida grows aerobically or anaerobically. The optimal growth temperature is $37^{\circ} \mathrm{C}$. The optimal $\mathrm{pH}$ range is $7.2-7.8$, but growth can occur in the range 6.2-9.0, depending upon composition of the medium. In liquid media, maximum growth is obtained in 16-24 hours. The broth becomes cloudy,

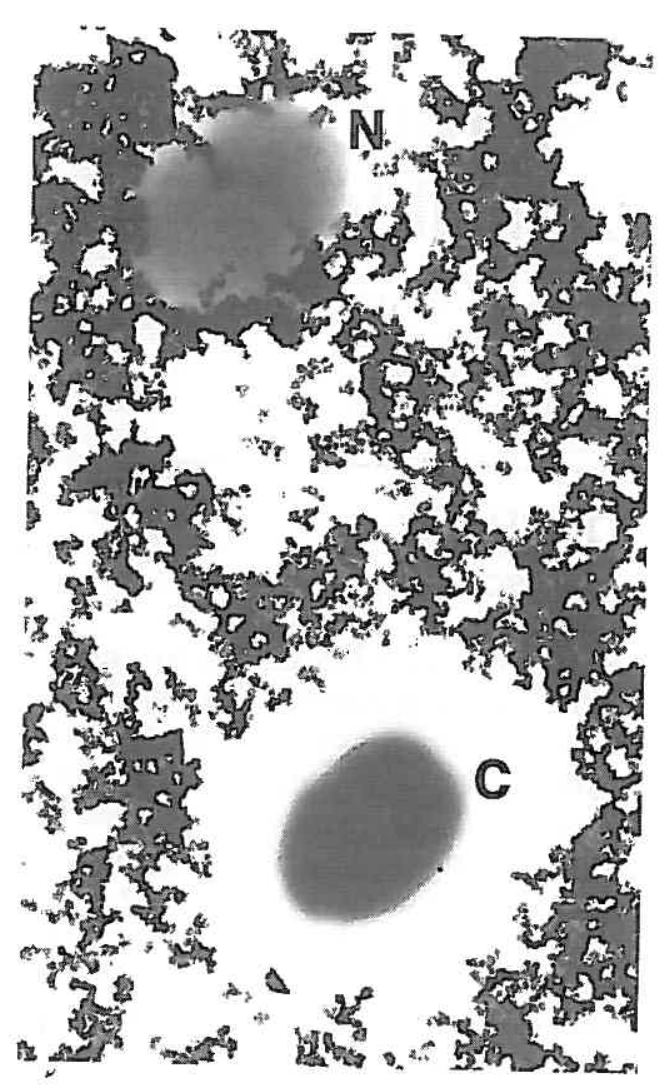

Figure 19.1. Electron photomicrograph of Pasteurella multocidaencapsulated cell $(C)$ and nonencapsulated cell $(N)$ suspended in India ink. $\times 19,000$.

and in a few days, a sticky sediment collects. With some isolates, a flocculent precipitate occurs.

The bacterium will grow on meat infusion media; growth is enhanced when the medium is enriched with peptone, casein hydrolysate, or avian serum. Blood or serum from some animals inhibits growth of $P$. mullocida. Inhibition is greatest from blood of horses, cattle, sheep, and goats; blood of chickens, ducks, swine, and water buffalo has little or no inhibitory action (152). Several selective media for isolation have been described $(27,28,44,99,111,160)$. Chemically defined media have been described by Jordan (92). Watko (167), Wessman and Wessman (169), and Flossmann et al. (42). Berkman (8) found that pantothenic acid and nicotinamide are essential for growth. Dextrose starch agar with $5 \%$ avian serum is an excellent medium for isolating and growing P. multocida.

\section{Colonial Morphology and Related Properties}

Colonial morphology observed with obliquely transmitted light is one of the most useful characteristics in the study of $P$. multocida. On primary isolation from birds with $\mathrm{FC}$, colonies may be iridescent, sectored with various intensities of iridescence, or blue with little or no iridescence (Figure 19.3). Iridescence is related to the presence of a capsule. The term 
FC and fowl typhoid or fowlpox were concurrently present, chloramphenicol treatment was not successful (81). A chloramphenicol-dexamethasone-pyribenzamine combination was used successfully with vaccination in treatment of FC in breeding turkeys. Respiratory problems, which occurred 1 week after the initial outbreak, responded readily to IM administration of this drug combination (51). Water-soluble erythromycin at the rate of $1 \mathrm{lb} / 50$ gallon of drinking water halted mortality in t2wo flocks of Muscovy ducklings infected with $P$. multocida (62). Fluoroquinolones are used successfully to treat FC. Pasteurella multocida isolates from poultry are typically highly susceptible to fluoroquinolones (47).

Antibiotics used in rations at very low levels for promotion of growth, according to the experiments of Dorsey and Harshfield (36), did not significantly influence the course of FC infection in inoculated birds. At therapeutic levels, birds that received penicillin and streptomycin in feed died at about the same rate as controls. No deaths occurred in groups that received sulfaquinoxaline or sulfamerazine. These workers also found oxytetracycline and chlortetracycline effective in preventing mortality in experimental FC in a small flock of laying birds; mortality was $80 \%$ in an untreated group compared with $12 \%$ in a group receiving mash containing oxytetracycline at the level of $500 \mathrm{~g} / \mathrm{ton}$. In 6 naturally occurring outbreaks, oxytetracycline at this level in feed checked mortality, but losses returned in 3 flocks after withdrawal of the antibiolic.

\section{Prevention and Control}

\section{Management Procedures}

Prevention of FC can be effected by eliminating reservoirs of P. multocida or by preventing their access to poultry flocks. Good management practices, with emphasis on sanitation, are the best means of preventing FC. Unlike many bacterial diseases, FC is not a disease of the hatchery. Therefore, infection occurs after birds are in the hands of the producer, and consideration must be given to the many ways that infection might be introduced into a flock.

The primary source of infection is usually sick birds or those that have recovered and still carry the causative organism. Only young birds should be introduced as new stock; they should be raised in a clean environment completely isolated from other birds. Isolation should be extended to housing. Unless separate houses can be provided for firstand second-year layer flocks, the older flock should be marketed in its entirety. Different species of birds should not be raised on the same premises. The danger of mixing birds from different flocks cannot be overemphasized. Farm animals (particularly pigs, dogs, and cats) should not have access to the poultry area. Water fountains should be self-cleaning, and feeders should be covered to prevent contamination as much as possible.

P. multocida has been recovered from many species of freeflying birds and warrants consideration as source of bacteria to poultry; wit measures should be taken to prevent their association with the flock. Raising turkeys in areas where FC is a serious problem may warrant their confinement in houses from which free-flying birds, rodents, and other animals can be excluded. If an outbreak of FC occurs, the flock should be quarantined and disposed of as soon as economically feasible. All housing and equipment should be cleaned and disinfected before repopulation.

\section{Vaccination}

Vaccination should be considered in areas where FC is prevalent, but it should not be substituted for good sanitary practice. Commercially produced bacterins and live vaccines are available. Bacterins usually contain whole cells of serotypes 1, 3, and 4 emulsified in an oil adjuvant. Because a bacterin will not provide protection against an FC challenge from a serotype not contained in that bacterin, an autogenous whole-cell bacterin containing a locally isolated strain other than serotypes 1,3 , of 4 may be used (63). The choice of adjuvant for an autogenous vaccine can be water-in-oil emulsion or aluminum hydroxide (9). Autogenous bacterins using aluminum hydroxide as the adjuvant are useful for the vaccination of turkey breeder or broiler breeder flocks that are in lay because the water-in-oil emulsion, in combination with the whole bacterial cell, results in a significant tissue response by the bird. This response can result in significant declines in egg production. The negative effect on egg production is less with 'aluminum hydroxide adjuvant whole-cell FC bacterins. It has beén well documented that aluminum hydroxide bacterins do not stimulate the immune response as well as water-in-oil bacterins $(69,107)$. Therefore, if an aluminum hydroxide bacterin is used, revaccination may be required to afford immunity to a flock for an entire laying cycle.

Three live vaccines available for use in the United States are $\mathrm{CU}$, a strain of low virulence; $\mathrm{M}-9$, a mutant of $\mathrm{CU}$ with very low virulence; and PM-1, a mutant of CU intermediate in virulence between $\mathrm{CU}$ and $\mathrm{M}-9$. Vaccination of chickens and turkeys with these live $P$. multocida vaccines induces protection against heterologous serotype challenge. The use of live FC vaccines stimulates an effective immune response but has the disadvantage of potentially resulting in mortality in the vaccinated birds (11). If the mortality post vaccination becomes excessive, it can be reduced by the administration of an antibiotic. This should be avoided, if possible, until at least 4 days post vaccination when there will be at least partial immunity induced by the vaccine (123).

When considering the most appropriate vaccination program for $\mathrm{FC}$, the following should be taken into consideration: prevalence of $\mathrm{FC}$ in the area, most prevalent serotypes of $P$. multocida in area, age of birds to be vaccinated, and the value of the birds to be vaccinated (i.e., breeder turkeys vs. commercial turkeys or parent chicken breeders vs. grandparent chicken breeders). There have been many successful vaccination protocols for chicken breeders against FC. Bacterins, live vaccines, or both are used, and usually 2 doses are given: the first at 8-10 weeks of age and the second at 18-20 weeks of age. Protection occurs only against serotypes contained in the 


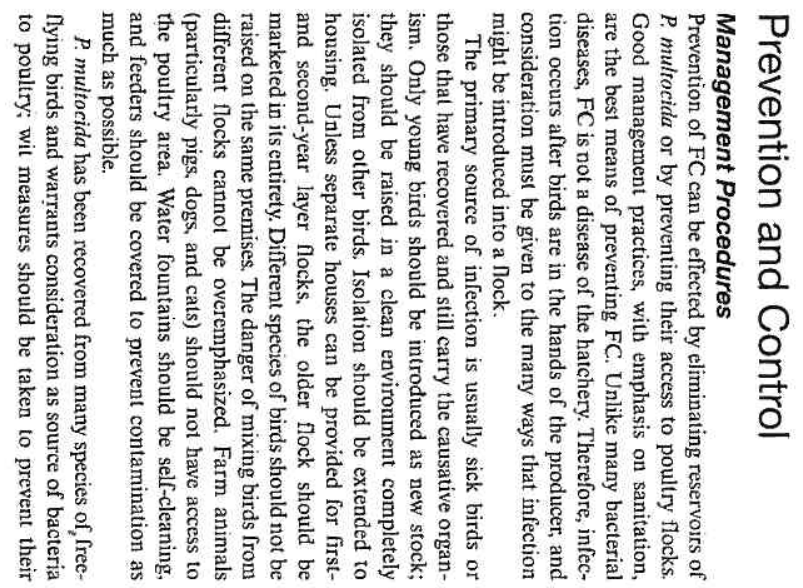

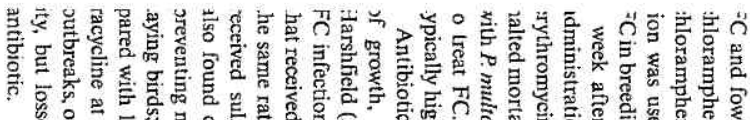

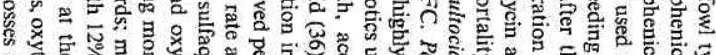

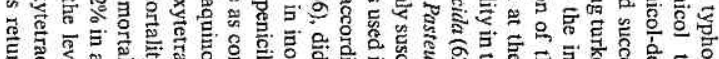

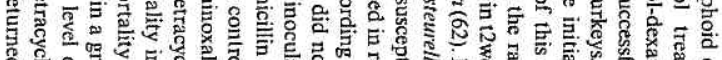

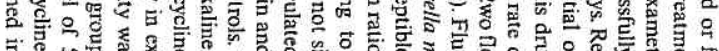

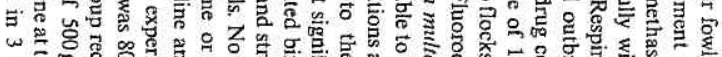

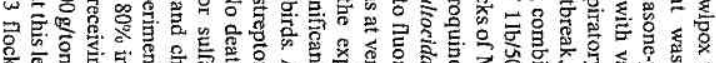

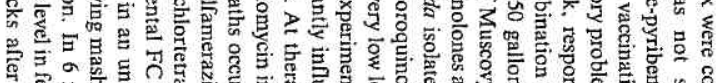

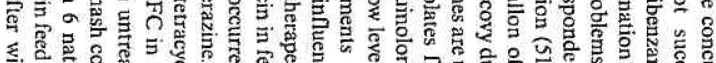

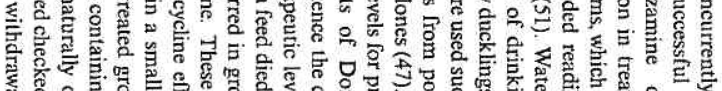

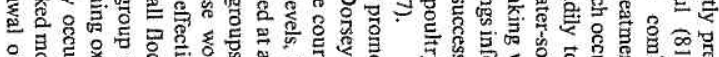

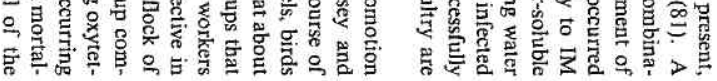

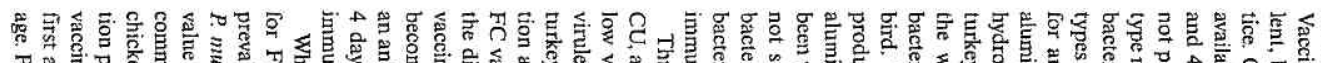

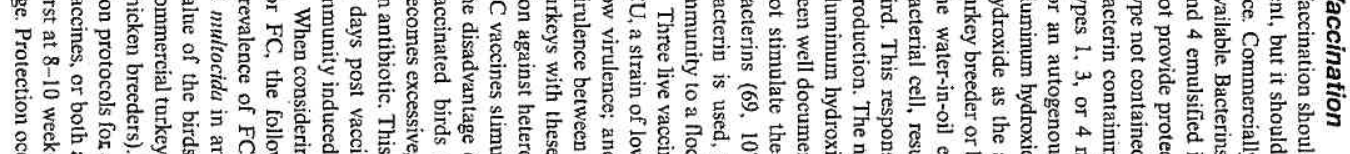

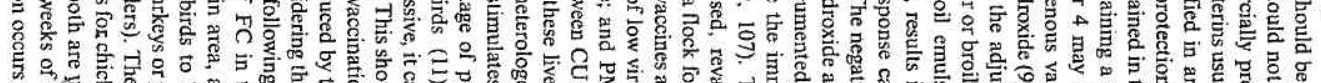

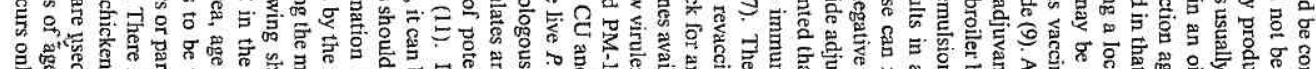

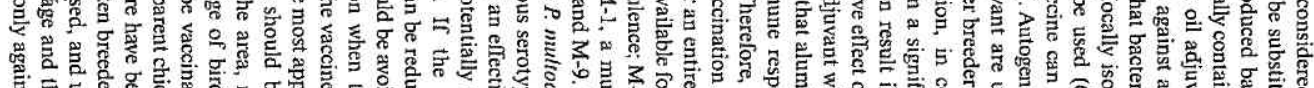

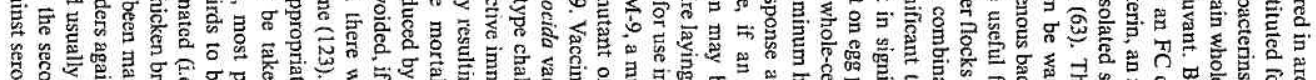

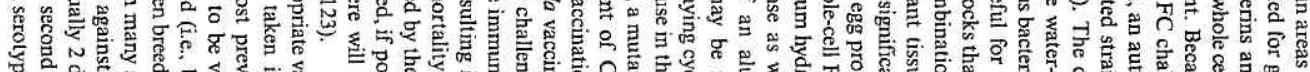

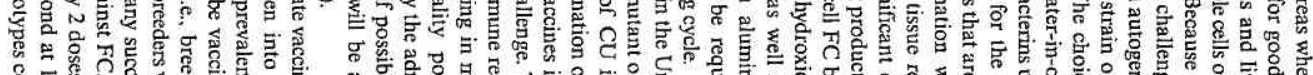

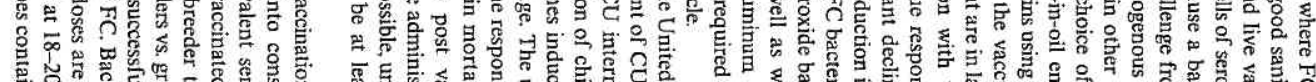

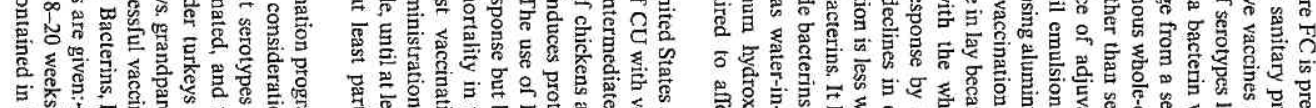

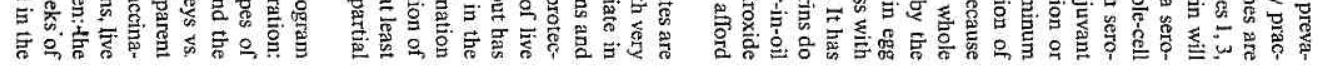

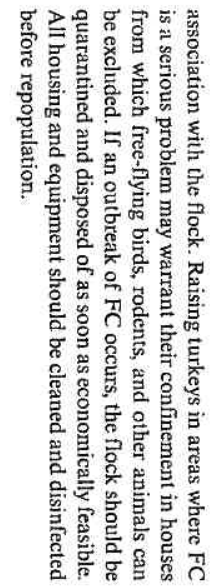

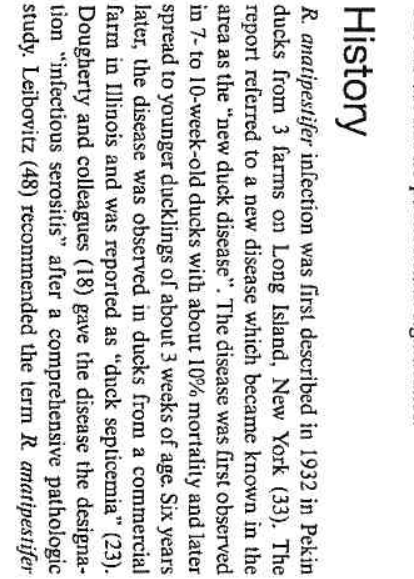

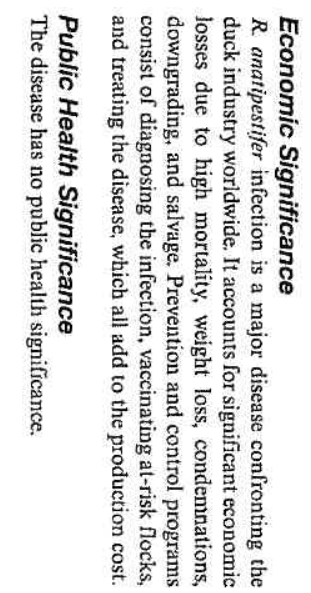

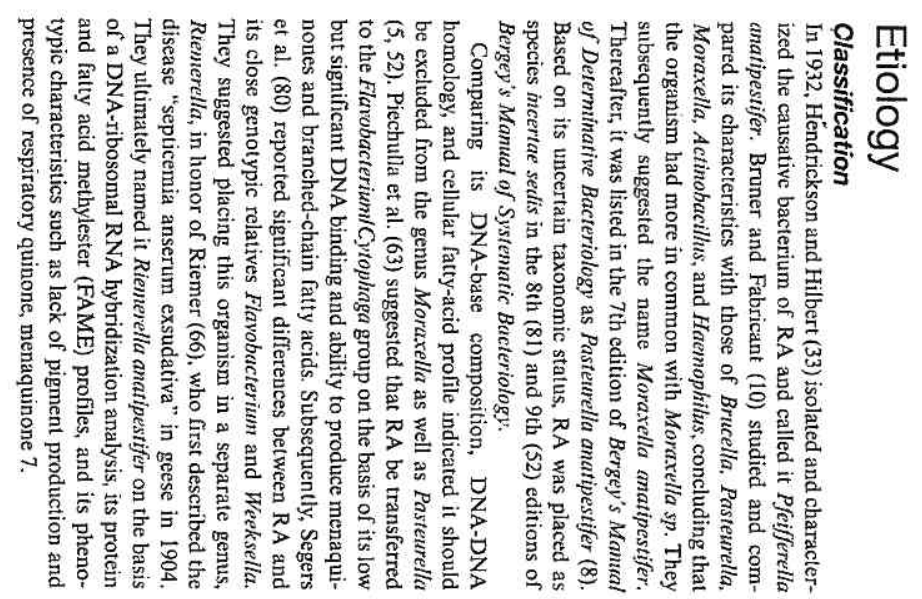

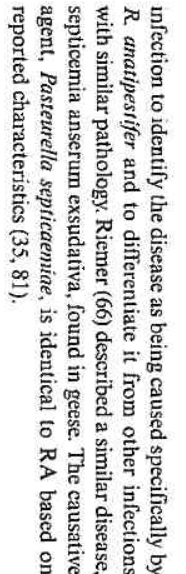

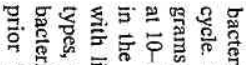

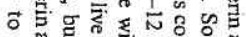

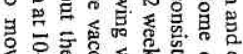

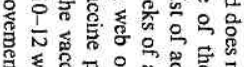

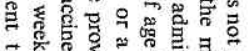

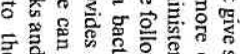

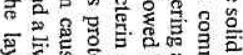

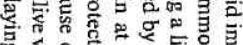

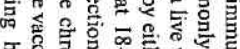

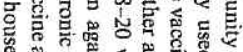

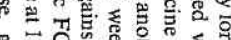

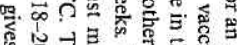

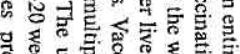

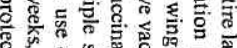

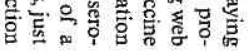

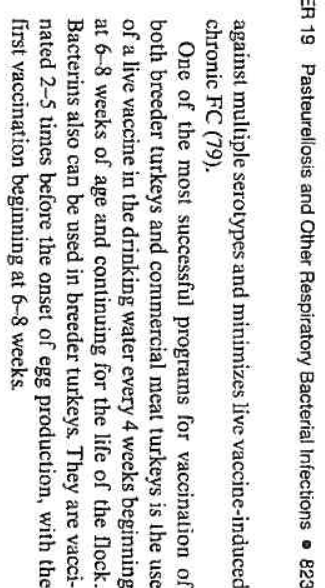


e Atso avalloblo 